\title{
Impact of Gestational Weight Gain on Perinatal Outcomes:Appropriateness of IOM Recommendations in Mainland China
}

\author{
Jing Hu \\ Peking Union Medical College Hospital \\ Jinsong Gao ( $\nabla$ gaojingsong@pumch.cn ) \\ Peking Union Medical College Hospital \\ Juntao Liu \\ Peking Union Medical College Hospital \\ Xietong Wang \\ Shandong Provincial Hospital \\ Jing He \\ Women's Hospital School Of Medicine Zhejiang University \\ Jingxia Sun \\ First Affiliated Hospital of Harbin Medical University \\ Xiaowei Liu \\ Beijing Obstetrics and Gynecology Hospital \\ Shixiu Liao \\ Henan Provincial People's Hospital \\ Shuang Liu \\ Chinese Academy of Medical Sciences \& Peking Union Medical College \\ Jing Dong \\ National Health and Family Planning Commission
}

\section{Research Article}

Keywords: Gestational Weight Gain, Perinatal Outcomes

Posted Date: December 1st, 2020

DOI: https://doi.org/10.21203/rs.3.rs-114855/v1

License: (c) (i) This work is licensed under a Creative Commons Attribution 4.0 International License. Read Full License 


\section{Abstract}

Abstract The study was conducted to evaluate the clinical feasibility of Institute of Medicine (IOM) recommendations on gestational weight gain (GWG) in mainland China. 88,297 singleton pregnancies from a nationwide birth registry study were included. GWG per week was calculated and grouped into within, below and above IOM (IOM) guidelines based on first trimester Chinese body weight index (BMI) status. Univariable and multivariable analyses were performed to determine the relationship between GWG category and perinatal outcomes. We found that excessive GWG was associated with increased risk in pregnancy induced hypertensive disorders (aOR 2.41, 95\% Cl 2.16-2.69), cesarean section (aOR 1.55, 95\% Cl 1.47-1.63 for nulliparas, aOR $1.51,95 \% \mathrm{Cl} 1.38-1.65$ for multiparas with no prior cesarean section), severe postpartum hemorrhage (aOR 1.15, 95\% $\mathrm{Cl} 1.06-1.26)$, large for gestational neonates (aOR1.76, 95\% $\mathrm{Cl} 1.69-1.85)$ and macrosomia (aOR 1.83, 95\% Cl 1.72-1.96), while inadequate GWG was correlated with higher risk in placenta abruption (aOR 1.54, 95\% Cl 1.29-1.85), fetal distress (aOR 1.19, 95\% Cl 1.12-1.26), and small for gestational neonates (aOR 1.50, 95\% Cl 1.41-1.60). Either GWG above or below was associated with increased risk in preterm birth (aOR 1.48, 95\% Cl 1.38-1.58 for above, aOR 1.47, 95\% Cl 1.31-1.64 for below), and neonatal asphyxia (aOR 2.28, 95\% Cl 2.00-2.61 for above, aOR 1.42, 95\% Cl 1.25-1.61 for below). GWG within IOM recommendations may help prevent various adverse perinatal outcomes and seemed suitable in Chinese population.

\section{Introduction}

Inappropriate GWG has remained to be a pronounced problem in both Western and Eastern countries. ${ }^{1,2,3}$ Insufficient weight gain has been linked with increased risk of poor fetal growth, ${ }^{1,4}$ preterm birth, ${ }^{1,5,6}$ and difficulty in establishing breastfeeding. ${ }^{7,8,9}$ While excessive GWG has been associated with a range of conditions, including gestational diabetes, ${ }^{10,11}$ pregnancy-induced hypertension, ${ }^{1,12,13,14}$ cesarean birth, ${ }^{1,15,16}$ and macrosomia. ${ }^{1,5,6,10}$ In 1970, the Institute of Medicine (IOM) reviewed the evidence concerning GWG that achieved optimal pregnancy outcomes, with revised versions published in 1990 and 2009. ${ }^{17,18}$ The latest version examined the trade offs between maternal and neonatal outcomes and GWG. In consideration that GWG has found to be differed by maternal pre-pregnancy weight status, ${ }^{19,20}$ the recommendations on GWG was based on WHO weight status criteria. ${ }^{21,22}$ In Jan 2018, the national prenatal care guideline for Chinese women was officially published, ${ }^{23}$ it accepted the IOM recommendations for Chinese with little sample sized evidence and ignored the difference between Westerners and Chinese in BMI characteristics. ${ }^{24}$ Chinese ethics possess a higher rate of body fat compared with Caucasian at the same $\mathrm{BMI},{ }^{25,26}$ therefore BMI cutoffs of $\geq 24$ for overweight and $\geq 28$ for obesity for Chinese population have been chosen based on the epidemiology evidence. ${ }^{27}$ Under different weight status classification system, some Chinese women would be classified as lower weight status and assigned to larger GWG targets. The appropriateness of recommending the IOM GWG guidelines for Chinese should be evaluated by large population evidence. We aim to assess the suitability of generalizing the IOM recommendations using localized BMI category criteria through evaluating the association between IOM GWG category and perinatal outcomes.

\section{Methods}




\section{Subjects}

Our study was performed in compliance with the Declaration of Helsinki (as revised in 2013). The retrospective birth registry study protocol was approved by the Ethics Committee of Peking Union Medical College Hospital (reference number PUMCH-JS-1151) under a waiver of informed consent from individual patients. Data were abstracted from the nationwide multicenter birth registry study carried out in 14 representative medical centers (including 2 secondary and 12 tertiary, 7 general hospitals, and 7 maternal and child health care centers) from 10 provinces in the four major economic regions of China from October 1 , 2016 to September 30,2017, Specially assigned clinicians at each hospital were responsible for collecting and reporting information of pregnant women who delivered after 20 weeks of gestation. Complete medical information for each birth, including maternal demographics, medical and obstetrical history, maternal and perinatal outcome information were retrospectively registered into a prospectively designed network-based standardized data acquisition system depending on digital and written form of medical records. The data acquisition system was prospectively designed with clear and detailed field definition by information center of National Research for Family Planning Institute. The accuracy and validity of data entered were reviewed and verified by a second researcher on a monthly basis. Data entered into the data collection system were ultimately exported from the central network-based database system.

Only ethically Chinese women over 18 years old with a singleton pregnancy who gave birth at 24 or more completed weeks of gestation were included. Women were eligible if they had a height, weight at delivery and either self-reported or recorded first trimester weight (11-14 gestational weeks), the later was mostly extracted from first trimester aneuploidy screening reports. Those with extreme values were excluded from analysis (maternal age at delivery older than 60 years old, height outside a range of 120-200 cm, first trimester weight lower than $25 \mathrm{~kg}$, GWG outside of a range of $-25-50 \mathrm{~kg}$ ). In this study, we have used first trimester BMI instead of pre-pregnancy BMI, in consideration that there has no reliable source of pre-pregnancy weight since most women came to obstetric units only after they became pregnant. BMI $\left(\mathrm{kg} / \mathrm{m}^{2}\right)$ was calculated as the ratio of the weight $(\mathrm{kg})$ at first trimester divided by height squared $\left(\mathrm{m}^{2}\right)$. GWG was calculated by weight before delivery minus weight recorded at first-trimester. In order to account for gestational age at delivery, we use average GWG per week for the second and the third trimester instead of total GWG. The WHO weight status criteria, the corresponding Chinese version and the associated IOM recommended GWG per week for the second and third trimester are listed in table1.

Table 1.IOM GWG recommendations for the second and the third trimester by pre-pregnancy weight status 


\begin{tabular}{|c|c|c|c|}
\hline $\begin{array}{l}\text { Weight } \\
\text { status }\end{array}$ & $\begin{array}{l}\text { WHO BMI category } \\
\text { criteria }\left(\mathrm{kg} / \mathrm{m}^{2}\right)^{21,22}\end{array}$ & $\begin{array}{l}\text { Chinese BMI category } \\
\text { criteria }\left(\mathrm{kg} / \mathrm{m}^{2}\right)^{27}\end{array}$ & $\begin{array}{c}\text { IOM-recommended weight gain } \\
\text { per week }(\mathrm{kg} / \text { week })^{18}\end{array}$ \\
\hline Underweight & a18.5 & $\square 18.5$ & $0.44-0.58$ \\
\hline $\begin{array}{l}\text { Normal } \\
\text { weight }\end{array}$ & $18.5-24.9$ & $\geq 18.5$ and $\square 24$ & $0.35-0.50$ \\
\hline Overweight & $25.0-29.9$ & $\geq 24$ and $\square 28$ & $0.23-0.33$ \\
\hline Obese & $\geq 30$ & $\geq 28$ & $0.17-0.27$ \\
\hline
\end{tabular}

\section{Perinatal outcomes}

Maternal outcomes included, pregnancy induced hypertensive disorders (PIH), including gestational hypertension, preeclampsia, HELLP (hemolysis elevated liver enzymes and low platelets syndrome), or eclampsia, premature rupture of membrane before onset of labor (PROM), cesarean section, severe postpartum hemorrhage (PPH, defined as estimated blood loss $\geq 1000 \mathrm{~mL}$ within 24 hours after delivery, or requires a blood transfusion, interventional or operational therapy for hemorrhage), placenta abruption, and operational vaginal birth. Occurrences of gestational diabetes mellitus (GDM, diagnosed using the oral glucose tolerance test at 24-28 weeks of pregnancy in accordance with 2013 WHO criteria ${ }^{28}$ ) were reported, but not evaluated as outcomes due to the nutritional counseling and dietary adjustments that occur after such a diagnosis and the consequent effect on GWG. Neonatal outcomes included, preterm birth (PTB), large for gestational age and small for gestational age (LGA and SGA) defined as birthweight above or below the 90th and 10th centiles of our local population standard chart after adjusting for gender and gestation, ${ }^{29}$ macrosomia (birth weight greater than $4000 \mathrm{~g}$ ), fetal distress was defined as either meconium stained amniotic fluid or persistent abnormal fetal heart rate, and newborn asphyxia defined as a postnatal Apgar score lower than 7 at five minute after birth. Preterm birth was considered for medically-indicated PTB and spontaneous PTB (SPTB), the later was further subdivided by whether premature rupture of membrane has occurred, namely, preterm premature rupture of membranes (PPROM) and SPTB without PROM.

\section{Statistical analyses}

Maternal demographics and characteristics were assessed and compared between individuals in different GWG categories using the Kruskal-Wallis test for continuous variables and the chi-square test for categorical variables. A multivariate logistic regression of the GWG categories and other variables was performed to estimate the adjusted odds ratio (OR) with $95 \% \mathrm{Cl}$ intervals of the maternal adverse outcomes and neonatal adverse outcomes whereby "within" IOM recommendation was reference. Multinomial logistics for analysis for the risk of each subgroup of preterm birth. Covariates included maternal age, conception method, parity, previous cesarean section, gestational diabetes, pre-gestational diabetes and chronic hypertension. Two- 
sided test alpha $=0.05$. Missing values processed censoring. All data analyses were performed using $\mathrm{R}$ version 4.0.2 (2020-06-22).

\section{Results}

A total of 99,960 women having delivery at or more than 20 completed weeks of gestation were identified in the national birth registry database, those non ethically Chinese, with missing data or implausible data concerning GWG or twin pregnancy were excluded. A total of 88,297 singleton pregnancies were therefore available for final analysis, only $0.2 \%$ of them presented in labor without prenatal care. Figure 1 summarizes the Flow chart for selection of eligible subjects.

Of the available 88,97 cases for analysis, $51,428(58.2 \%)$ were nulliparas. The median of maternal age at delivery was 30 years (IQR: $28-34) .71 .7 \%(n=63,351)$ of the women were normal weight in first trimester, less than half (44.1\%) of the total population gained within the IOM guidelines, $36.5 \%$ and $19.4 \%$ gained below and above the guidelines, respectively. Between the three GWG categories, there were differences in maternal age, parity, baseline BMI, conception method, prior cesarean birth, chronic hypertension and pregestational diabetes, ( $\mathrm{p} \otimes 0.001$ for all comparisons).(maternal demographic and characteristics information are listed in Table 2)

Table 2.Maternal demographic and characteristics 


\begin{tabular}{|c|c|c|c|c|c|}
\hline \multirow[t]{2}{*}{ Characteristics } & \multicolumn{4}{|c|}{ GWG } & \multirow[t]{2}{*}{ p-value } \\
\hline & All & Below & Within & Above & \\
\hline $\mathbf{n}$ & 88,297 & $32,210(36.5)$ & $38,935(44.1)$ & $17,152(19.4)$ & \\
\hline Maternal age(years) & $30.00(28,34)$ & $30(27,34)$ & $30(28,34)$ & $30(28,34)$ & $<0.001$ \\
\hline$\geq 16$ and $<20$ & $349(0.4)$ & $157(0.5)$ & $139(0.4)$ & $53(0.3)$ & \\
\hline $20 \geq$ and $<25$ & $5,459$ ( 6.2$)$ & $2,131(6.6)$ & $2,338(6.0)$ & $990(5.8)$ & \\
\hline $25 \geq$ and $<30$ & $33,709(38.2)$ & $12,289(38.2)$ & $15,063(38.7)$ & $6,357(37.1)$ & \\
\hline $30 \geq$ and $<35$ & $30,540(34.6)$ & $11,308(35.1)$ & $13,349(34.3)$ & $5,883(34.3)$ & \\
\hline $35 \geq$ and $<40$ & $15,087(17.1)$ & $5,253(16.3)$ & $6,642(17.1)$ & 3,192(18.6) & \\
\hline$\geq 40$ & 3,153 ( 3.6) & $1,072(3.3)$ & $1,404(3.6)$ & $6,77(3.9)$ & \\
\hline Prenatal examination & 88,077 (99.8) & $32,131(99.8)$ & $38,835(99.7)$ & $17,111(99.8)$ & 0.913 \\
\hline Height,cm & $160.0(158.0,165.0)$ & $160.0(158.0,164.0)$ & $160.0(158.0,165.0)$ & $162.0(160.0,165.0)$ & $<0.001$ \\
\hline First trimester weight,kg & $53.0(49.0,59.0)$ & $51.0(46.5,56.0)$ & $53.0(49.5,58.0)$ & $59.6(52.0,65.0)$ & $<0.001$ \\
\hline First trimester BMI $\left(\mathrm{kg} / \mathrm{m}^{2}\right)$ & $20.50(18.9,22.5)$ & $19.80(18.1,21.7)$ & $20.40(19.1,22.1)$ & $22.40(19.9,24.9)$ & $<0.001$ \\
\hline $\mathrm{BMI}<18.5$ & $17,459(19.8)$ & $10,648(33.1)$ & $5,449(14.0)$ & $1,362(7.9)$ & \\
\hline $18.5 \leq \mathrm{BMI}<24$ & $63351(71.7)$ & 20537(63.8) & 31244(80.2) & 11570(67.5) & \\
\hline $24 \leq \mathrm{BMI}<28$ & $5580(6.3)$ & $762(2.4)$ & $1735(4.5)$ & $3083(18.0)$ & \\
\hline $\mathrm{BMI} \geq 28$ & 1907 ( 2.2) & 263(0.8) & $507(1.3)$ & $1137(6.6)$ & \\
\hline Total GWG, kg & $15.00(12.00,18.00])$ & $12.00(10.0,13.0)$ & $16.00(15.0,18.0)$ & $20.00(18.0,24.0)$ & $<0.001$ \\
\hline GWG per week, kg/week & $0.38(0.31,0.46)$ & $0.30(0.25,0.33)$ & $0.41(0.37,0.46)$ & $0.53(0.46,0.61)$ & $<0.001$ \\
\hline In vitro fertilization & $1,780(2.0)$ & $691(2.1)$ & $704(1.8)$ & $385(2.2)$ & $<0.001$ \\
\hline Nulliparas & $51,428(58.2)$ & $19,355(60.1)$ & $22,308(57.3)$ & $9,765(56.9)$ & $<0.001$ \\
\hline GA at delivery, $d$ & $272.8(13.0)$ & $272.5(13.5)$ & 273.7(11.9) & $271.3(14.4)$ & $<0.001$ \\
\hline Pre-existing diabetes & $385(0.4)$ & $181(0.6)$ & $114(0.3)$ & $90(0.5)$ & $<0.001$ \\
\hline Chronic hypertension & $372(0.4)$ & $99(0.3)$ & $125(0.3)$ & $148(0.9)$ & $<0.001$ \\
\hline
\end{tabular}




\begin{tabular}{|c|c|c|c|c|c|}
\hline Cesarean section history & $16511(18.7)$ & $5548(17.2)$ & 7289(18.7) & $3674(21.4)$ & $<0.001$ \\
\hline Gestational diabetes & $11,196(12.7)$ & $4,232(13.1)$ & $4,514(11.6)$ & $2,450(14.3)$ & $<0.001$ \\
\hline Cesarean section & $38,181(43.2)$ & $12,297(38.2)$ & $16,757(43.0)$ & $9,127(53.2)$ & $<0.001$ \\
\hline Repeat Cesarean section & $15,780(95.6 \%)$ & 5,212 (93.9) & $7,013(96.2)$ & $3,555(96.8)$ & $<0.001$ \\
\hline
\end{tabular}

Abbreviations: GA gestational age

Data are presented as either number and percentage (\%) for categorical variables, median and inter-quartile range for continuous non normal variables, mean and standard deviation (SD) for continuous normal variables.

\section{Effects of GWG on maternal outcomes}

For maternal outcomes, compared to GWG within recommendation, GWG above was associated with higher risk in cesarean birth, both in nulliparas $(45.0 \%$ vs $34.3 \%$, aOR $1.55,95 \% \mathrm{Cl} 1.47-1.63)$ and multiparas with no prior cesarean section $(31.7 \%$ vs $22.4 \%$, aOR $1.51,95 \% \mathrm{Cl} 1.38-1.65)$, PIH $(9.0 \%$ vs $3.7 \%$, aOR $2.41,95 \% \mathrm{Cl}$ 2.16-2.69) and severe postpartum hemorrhage (4.8\% vs $4.1 \%$, aOR $1.15,95 \% \mathrm{Cl} 1.06-1.26)$. While inadequate GWG was linked with higher risk in PROM (16\% vs $14 \%$, aOR $1.15,95 \% \mathrm{Cl} 1.11-1.20)$, and placenta abruption (0.86\% vs $0.56 \%$, aOR $1.54,95 \% \mathrm{Cl} 1.29-1.85)$ (listed in Table 3 ,Figure 2a and Figure3a )

Table 3. Maternal Outcomes According to the IOM GWG Categories 


\begin{tabular}{|c|c|c|c|c|c|c|c|}
\hline \multirow[t]{3}{*}{ :omes } & \multicolumn{7}{|c|}{ GWG } \\
\hline & \multicolumn{3}{|c|}{ Below } & \multirow{2}{*}{$\begin{array}{l}\text { Within } \\
\text { N (\%) }\end{array}$} & \multicolumn{3}{|c|}{ Above } \\
\hline & N (\%) & Unadjusted OR,p-value & Adjusted OR,p-value & & N (\%) & $\begin{array}{l}\text { Unadjusted } \\
\text { OR,p-value }\end{array}$ & $\begin{array}{l}\text { Adjusted OR,p- } \\
\text { value }\end{array}$ \\
\hline $\begin{array}{l}\text { tensive } \\
\text { lers of } \\
\text { Iancy* }\end{array}$ & $\begin{array}{c}784 \\
(2.4 \%)\end{array}$ & $0.65(0.59,0.71) p<0.001$ & $0.63(0.57,0.68) p<0.001$ & $\begin{array}{l}1,445 \\
(3.7 \%)\end{array}$ & $\begin{array}{l}1,544 \\
(9.0 \%)\end{array}$ & $\begin{array}{c}2.57(2.38,2.76) \\
p<0.001\end{array}$ & $\begin{array}{c}2.44(2.26,2.64) \\
p<0.001\end{array}$ \\
\hline iM† & $4,232(13.14 \%)$ & $1.15(1.10,1.21) p<0.001$ & $\begin{array}{c}1.16(1.11,1.22) \\
p<0.001\end{array}$ & $4,514(11.6 \%)$ & $2,450(14.3 \%)$ & $\begin{array}{c}1.27(1.20,1.34) \\
p<0.001\end{array}$ & $\begin{array}{c}1.24(1.17,1.31) \\
p<0.001\end{array}$ \\
\hline $\mathrm{JM}^{*}$ & $5,159(16.0 \%)$ & $\begin{array}{c}1.17(1.12,1.22) \\
p<0.001\end{array}$ & $1.15(1.11,1.20) p<0.001$ & $5,456(14.0 \%)$ & $2,226(13.0 \%)$ & $\begin{array}{c}0.92(0.87,0.96) \\
p=0.001\end{array}$ & $\begin{array}{c}0.92(0.87,0.97) \\
p=0.001\end{array}$ \\
\hline $\begin{array}{l}\text { arean } \\
\text { on for } \\
\text { aras㧊 }\end{array}$ & $\begin{array}{c}5,679 \\
(29.3 \%)\end{array}$ & $0.80(0.76,0.83) p<0.001$ & $0.76(0.73,0.80) p<0.001$ & $\begin{array}{c}7,649 \\
(34.3 \%)\end{array}$ & $\begin{array}{r}4,395 \\
(45.0 \%)\end{array}$ & $\begin{array}{c}1.57(1.49,1.65) \\
p<0.001\end{array}$ & $\begin{array}{c}1.55(1.47,1.63) \\
p<0.001\end{array}$ \\
\hline $\begin{array}{l}\text { arean } \\
\text { on for } \\
\text { iparas } \\
\text { sut CS } \\
\text { rył } \neq\end{array}$ & $\begin{array}{c}1,406 \\
(19.2 \%)\end{array}$ & $0.82(0.76,0.89) p<0.001$ & $0.82(0.76,0.89) p<0.001$ & $7,243(22.4 \%)$ & $\begin{array}{c}2,536 \\
(31.7 \%)\end{array}$ & $\begin{array}{c}1.61(1.47,1.75) \\
p<0.001\end{array}$ & $\begin{array}{c}1.51(1.38,1.65) \\
\mathrm{p}<0.001\end{array}$ \\
\hline $\begin{array}{l}\text { arean } \\
\text { ion on } \\
\text { ernal } \\
\text { uest* }\end{array}$ & $\begin{array}{l}3,099 \\
(9.6 \%)\end{array}$ & $1.11(1.06,1.17) \mathrm{p}<0.001$ & $1.08(1.02,1.14) \mathrm{p}<0.001$ & $\begin{array}{l}3,398 \\
(8.7 \%)\end{array}$ & $\begin{array}{c}2,031 \\
(11.8 \%)\end{array}$ & $\begin{array}{c}1.40(1.32,1.49) \\
p<0.001\end{array}$ & $\begin{array}{c}1.37(1.29,1.45) \\
p<0.001\end{array}$ \\
\hline $\begin{array}{l}\text { vartum } \\
\text { rhage§ }\end{array}$ & $\begin{array}{l}1,276 \\
(4.0 \%)\end{array}$ & $0.96(0.89,1.04) \mathrm{p}=0.32$ & $0.96(0.89,1.04) \mathrm{p}=0.32$ & $\begin{array}{l}1,600 \\
(4.1 \%)\end{array}$ & $\begin{array}{c}817 \\
(4.8 \%)\end{array}$ & $\begin{array}{c}1.17(1.07,1.27) \\
p<0.001\end{array}$ & $\begin{array}{c}1.15(1.06,1.26) \\
p=0.002\end{array}$ \\
\hline $\begin{array}{l}\text { h prior } \\
\text { 'S }\end{array}$ & $259(4.7 \%)$ & $1.10(0.92,1.30) \mathrm{p}=0.29$ & $1.12(0.94,1.32) \mathrm{p}=0.21$ & $312(4.3 \%)$ & $194(5.3 \%)$ & $\begin{array}{c}1.25(1.04,1.50) \\
p=0.019\end{array}$ & $\begin{array}{c}1.27(1.05,1.52) \\
p=0.012\end{array}$ \\
\hline $\begin{array}{l}\text { thout } \\
\text { r CS }\end{array}$ & $1,017(3.8 \%)$ & $0.94(0.86,1.02) \mathrm{p}=0.12$ & $0.92(0.85,1.01) \mathrm{p}=0.070$ & $1,288(4.1 \%)$ & $623(4.6 \%)$ & $\begin{array}{c}1.14(1.04,1.26) \\
p=0.008\end{array}$ & $\begin{array}{c}1.12(1.01,1.24) \\
p=0.025\end{array}$ \\
\hline :enta & $277(0.86 \%)$ & $1.55(1.30,1.85) \mathrm{p}$ & $1.54(1.30,1.85)$ & $217(0.56 \%)$ & $121(0.71 \%)$ & 1.27 (1.01, & 1.21(0.96, \\
\hline
\end{tabular}




\begin{tabular}{|c|c|c|c|c|c|c|c|}
\hline ption* & & $<0.001$ & $p<0.001$ & & & $\begin{array}{c}\text { 1.58) } \\
\mathrm{p}=0.037\end{array}$ & 1.51) $\mathrm{p}=0.098$ \\
\hline $\begin{array}{l}\text { ational } \\
\text { jinal } \\
\text { th§ }\end{array}$ & $725(3.6 \%)$ & $1.09(0.98,1.21) \mathrm{p}=0.10$ & $1.03(0.93,1.14) \mathrm{p}=0.59$ & $743(3.4 \%)$ & $219(2.7 \%)$ & $\begin{array}{c}\text { 0.81(0.69, } \\
\text { 0.94) } \mathrm{p}=0.007\end{array}$ & $\begin{array}{c}0.79(0.68 \\
0.92) \mathrm{p}=0.004\end{array}$ \\
\hline
\end{tabular}

Abbreviations: OR, odds ratio; CI, Confidence interval; GDM, gestational diabetes; PROM, premature rupture of membrane; CS, Cesarean section

*The analysis was adjusted for maternal age, conception method, parity, chronic hypertension, pre-gestational diabetes, and gestational diabetes.

†The analysis was adjusted for maternal age, conception method, parity, chronic hypertension, previous cesarean section history and pre-gestational diabetes.

¥ The analysis was adjusted for maternal age, conception method, chronic hypertension, pre-gestational diabetes, and gestational diabetes.

§The analysis was adjusted for maternal age, conception method, parity, chronic hypertension, prior cesarean section, pregestational diabetes.

The bolded values indicate statistical significance.

\section{Effects of GWG on neonatal outcomes}

For the neonatal outcomes, GWG outside the IOM guidelines was associated with overall PTB $(10.0 \%$ for above, aOR 1.48, 95\% Cl 1.38-1.58, 8.1\% for below, aOR 1.22, 95\% $\mathrm{Cl} 1.15-1.29)$ ), both in indicated (3.6\% for above, aOR $2.04,95 \% \mathrm{Cl} 1.82-2.29,2.1 \%$ for below, aOR $1.27,95 \% \mathrm{Cl} 1.14-1.42)$ and spontaneous PTB (6.4\% for above, aOR1.25, 95\% Cl 1.16-1.35, 6.0\% for below, aOR 1.19, 95\% Cl 1.11-1.27) compared with GWG within the IOM guidelines ( $6.7 \%$ for overall, $1.7 \%$ for medically-indicated PTB and $5.0 \%$ for spontaneous PTB), it was also associated with newborn asphyxia (2.81\% for above, aOR 2.28, 95\% Cl 2.00-2.61, 1.73\% for below, aOR $1.42,95 \% \mathrm{Cl} 1.25-1.61,1.22 \%$ for within). GWG above the guidelines was associated with large for gestational age neonates $(36.6 \%$ for above, $16.9 \%$ for within, aOR1.76, $95 \% \mathrm{Cl} 1.69-1.85)$ and macrosomia $(11.0 \%$ for above, $6.3 \%$ for within, aOR $1.83,95 \% \mathrm{Cl} 1.72-1.96$ ). While GWG below the guidelines was associated with fetal distress (8.5\% for below,7.1\% for within, aOR 1.19, 95\% Cl 1.12-1.26) and small for gestational neonates (8.5\% for below, $5.8 \%$ for within, aOR 1.50, 95\% Cl 1.41-1.60).(listed in Table 4 ,Figure 2b and Figure $3 \mathrm{~b}$ )

Table 4.Neonatal Outcomes According to the IOM GWG Categories 


\begin{tabular}{|c|c|c|c|c|c|c|c|}
\hline \multirow[t]{3}{*}{ Outcomes } & \multicolumn{7}{|c|}{ GWG } \\
\hline & \multicolumn{3}{|c|}{ Below } & \multirow{2}{*}{$\begin{array}{l}\text { Within } \\
\text { N (\%) }\end{array}$} & \multicolumn{3}{|c|}{ Above } \\
\hline & N (\%) & $\begin{array}{l}\text { Unadjusted } \\
\text { OR, p-value }\end{array}$ & Adjusted OR, p-value & & $\mathrm{N}(\%)$ & $\begin{array}{l}\text { Unadjusted } \\
\text { OR, p-value }\end{array}$ & Adjusted OR, p-value \\
\hline $\begin{array}{c}\text { Preterm } \\
\text { birth }\end{array}$ & $2,601(8.1 \%)$ & $\begin{array}{c}1.22(1.15,1.28) \\
p<0.001\end{array}$ & $1.22(1.15,1.29) p<0.001$ & $2,626(6.7 \%)$ & $1,712(10.0 \%)$ & $\begin{array}{c}1.53(1.44,1.63) \\
p<0.001\end{array}$ & $1.48(1.38,1.58) p<0.001$ \\
\hline 028W & $90(0.28 \%)$ & $\begin{array}{c}3.15(2.13,4.66) \\
p<0.001\end{array}$ & $3.28(2.19,4.90) p<0.001$ & $35(0.09 \%)$ & $36(0.21 \%)$ & $\begin{array}{c}2.42(1.52,3.85) \\
p<0.001\end{array}$ & $2.41(1.50,3.87) \mathrm{p}<0.001$ \\
\hline $28 \sim 32 W$ & $447(1.4 \%)$ & $\begin{array}{c}1.52(1.32,1.75) \\
p<0.001\end{array}$ & $1.54(1.34,1.78) p<0.001$ & $361(0.93 \%)$ & $336(2.0 \%)$ & $\begin{array}{c}2.19(1.88,2.54) \\
p<0.001\end{array}$ & $2.07(1.78,2.42) p<0.001$ \\
\hline $32 \sim 734 \mathrm{~W}$ & $339(1.0 \%)$ & $\begin{array}{c}1.15(0.99,1.34) \\
p=0.063\end{array}$ & $1.15(0.99,1.34) p=0.070$ & $361(0.93 \%)$ & $285(1.7 \%)$ & $\begin{array}{c}1.86(1.59,2.17) \\
p<0.001\end{array}$ & $1.78(1.52,2.09) \mathrm{p}<0.001$ \\
\hline $34 \sim 737 W$ & $1,725(5.4 \%)$ & $\begin{array}{c}1.13(1.06,1.21) \\
p<0.001\end{array}$ & $1.13(1.06,1.21) p<0.001$ & $1,869(4.8 \%)$ & $1,055(6.2 \%)$ & $\begin{array}{c}1.33(1.23,1.44) \\
p<0.001\end{array}$ & $1.28(1.19,1.39) \mathrm{p}<0.001$ \\
\hline $\begin{array}{c}\text { Indicated } \\
\text { preterm } \\
\text { birth }\end{array}$ & $670(2.1 \%)$ & $\begin{array}{c}1.24(1.11,1.38) \\
p<0.001\end{array}$ & $1.27(1.14,1.42) \mathrm{p}<0.001$ & $658(1.7 \%)$ & $616(3.6 \%)$ & $\begin{array}{c}2.17(1.94,2.42) \\
p<0.001\end{array}$ & $2.04(1.82,2.29) \mathrm{p}<0.001$ \\
\hline $\begin{array}{l}\text { Spontaneous } \\
\text { preterm } \\
\text { birth }\end{array}$ & 1,931(6.0\%) & $\begin{array}{c}1.20(1.12,1.28) \\
p<0.001\end{array}$ & $1.19(1.11,1.27) p<0.001$ & $1,968(5.0 \%)$ & $1,096(6.4 \%)$ & $\begin{array}{c}1.28(1.19,1.38) \\
p<0.001\end{array}$ & $1.25(1.16,1.35) \mathrm{p}<0.001$ \\
\hline $\begin{array}{l}\text { a.with } \\
\text { PROM }\end{array}$ & $711(2.2 \%)$ & $\begin{array}{c}1.39(1.24,1.54) \\
p<0.001\end{array}$ & $1.35(1.21,1.50) \mathrm{p}<0.001$ & $624(1.6 \%)$ & $377(2.2 \%)$ & $\begin{array}{c}1.38(1.21,1.57) \\
p<0.001\end{array}$ & $1.35(1.18,1.53) p<0.001$ \\
\hline $\begin{array}{c}\text { b.without } \\
\text { PROM }\end{array}$ & 1,220(3.8\%) & $\begin{array}{c}1.10(1.02,1.19) \\
p=0.017\end{array}$ & $1.10(1.02,1.20) \mathrm{p}=0.016$ & 1,344(3.4\%) & $719(4.2 \%)$ & $\begin{array}{c}1.22(1.11,1.34) \\
p<0.001\end{array}$ & $1.19(1.09,1.31) \mathrm{p}<0.001$ \\
\hline LGA & $3,461(11.2 \%)$ & $\begin{array}{c}0.62(0.59,0.65) \\
p<0.001\end{array}$ & $0.61(0.59,0.64) p<0.001$ & $6,249(16.9 \%)$ & $4,322(36.6 \%)$ & $\begin{array}{c}1.78(1.71,1.87) \\
p<0.001\end{array}$ & $1.76(1.69,1.85) \mathrm{p}<0.001$ \\
\hline Macrosomia & $1066(3.5 \%)$ & $\begin{array}{c}0.54(0.50,0.58) \\
p<0.001\end{array}$ & $0.53(0.49,0.57) \mathrm{p}<0.001$ & $2316(6.3 \%)$ & $1782(11.0 \%)$ & $\begin{array}{c}1.85(1.73,1.97) \\
p<0.001\end{array}$ & $1.83(1.72,1.96) \mathrm{p}<0.001$ \\
\hline SGA & $2,619(8.5 \%)$ & $1.52(1.43,1.61)$ & $1.50(1.41,1.60) p<0.001$ & $2,128(5.8 \%)$ & $748(4.6 \%)$ & $0.79(0.73,0.86)$ & $0.78(0.72,0.86) p<0.001$ \\
\hline
\end{tabular}




\begin{tabular}{|c|c|c|c|c|c|c|c|}
\hline & \multicolumn{3}{|c|}{$\mathrm{p}<0.001$} & & \multicolumn{3}{|c|}{$\mathrm{p}<0.001$} \\
\hline $\begin{array}{c}\text { Fetal } \\
\text { distress }\end{array}$ & $2,739(8.5 \%)$ & $\begin{array}{c}1.22(1.16 \\
1.29) p<0.001\end{array}$ & $\begin{array}{c}1.19(1.12,1.26) \\
p<0.001\end{array}$ & $2,749(7.1 \%)$ & $995(5.8 \%)$ & $\begin{array}{c}0.81(0.75 \\
0.87) p<0.001\end{array}$ & $\begin{array}{c}0.80(0.74,0.86) \\
p<0.001\end{array}$ \\
\hline $\begin{array}{l}\text { Newborn } \\
\text { asphyxia }\end{array}$ & $530(1.73 \%)$ & $\begin{array}{c}1.42(1.25,1.61) \\
p<0.001\end{array}$ & $1.42(1.25,1.61) p<0.001$ & $451(1.22 \%)$ & $454(2.81 \%)$ & $\begin{array}{c}2.33(2.04,2.66) \\
p<0.001\end{array}$ & $2.28(2.00,2.61) p<0.001$ \\
\hline
\end{tabular}

Abbreviations: OR,odds ratio; CI, Confidence interval; PROM, Premature rupture of membrane; LGA, large for gestational age; SGA, small for gestational age.

The analysis was adjusted for maternal age, parity, conception method, chronic hypertension, prior cesarean section, pregestational diabetes, and gestational diabetes.

The bolded values indicate statistical significance.

\section{Discussion}

In this analysis of 88,297 singleton pregnancies, we found associations between not achieving gestational weight gain goals recommended by IOM and several adverse maternal and neonatal outcomes including cesarean birth, PROM, PIH, postpartum hemorrhage, PTB, LGA, SGA, macrosomia, fetal distress and newborn asphyxia.

Our findings corresponds to other studies including a recent meta analysis of GWG and pregnancy outcomes that included 1,309,136 women. ${ }^{1}$ Specifically, the study showed that insufficient GWG was related to SGA (OR $1.53,95 \% \mathrm{Cl} 1.44-1.64)$ and preterm birth (OR 1.70, 95\% CI 1.32-2.20), whereas excessive GWG was associated with LGA (OR 1.85, 95\%Cl 1.76-1.95), macrosomia (OR 1.95, 95\% Cl 1.79-2.11), and cesarean birth (OR 1.30, $95 \% \mathrm{Cl} 1.25-1.35)$. A similar study from the US including 29,861 pregnancies also evaluated the relationship between excessive GWG and PIH (aOR 1.84, 95\% Cl 1.66-2.04), the prevalence of GDM in the total population was $6.2 \% .{ }^{16}$ However, we found in our study that the impact of excessive GWG on the risk of PIH in Chinese seems to be larger than in the Westerners (aOR 2.44, 95\% Cl 2.26-2.64), and the prevalence of GDM was $12.7 \%$. Although it is not possible to perform statistical comparison based on these figures. As it is well known that body fat percentage in Chinese is higher than that in Caucasian at the same GWG category, the metabolic mechanism leading to PIH and GDM may manifest that a lower cutoff at $24 \mathrm{~kg} / \mathrm{m}^{2}$ for defining overweight and $28 \mathrm{~kg} / \mathrm{m}^{2}$ for defining obese in Chinese would be more applicable for risk assessment.

In addition to common perinatal outcomes associated with poor GWG control and well described in previous studies, we also explored the possible relationship with premature rupture of membrane (PROM) and placenta abruption. In particular, inadequate GWG was associated with higher risk in PROM (aOR 1.15, 95\% Cl 1.111.20) and placenta abruption (aOR $1.54,95 \% \mathrm{Cl} 1.29-1.85)$, indicating that nutritional factors or underlying social economic factors may play a role in the pathogenesis of these conditions. ${ }^{30}$ Considerably fewer 
studies have evaluated the specific correlation between GWG and preterm birth subgroup (medically-indicated PTB, PPROM and SPTB without PROM) and gestational week distribution. ${ }^{31,32,33}$ In our study, we found that excessive GWG had greater influence on total PTB than insufficient GWG (aOR 1.48, 95\% CI 1.38-1.58 vs aOR $1.22,95 \% \mathrm{Cl} 1.15-1.29$ ), with highest odds ratio in medically-indicated PTB subgroup (aOR 2.04, 95\% CI 1.822.29), while insufficient GWG had highest odds ratio in PPROM subgroup (aOR 1.35, 95\% CI 1.21-1.50), and among gestational weeks between 24 and 28 (aOR 3.28, 95\%Cl 2.19-4.90). Such findings is different from similar population based studies from developed countries but supports study from middle income countries. ${ }^{1,5,34}$

Studies testifying IOM recommendations among Chinese have reached inconclusive results, ${ }^{23,35,36}$ Single site study including 4,736 term pregnancies using the WHO BMI cut-off points, concluded that IOM GWG recommendations are appropriate for Chinese women. ${ }^{23}$ While study carried out in Hong Kong including 754 pregnancies with complete anthropometry data, ${ }^{35}$ found that the IOM recommended weight gain ranges are narrower than observed. However, larger cohort study encompassing 13,717 pregnancies with optimal outcomes conducted in a Middle-South city of China revealed that the IOM recommended GWG ranges are still within the middle $70 \%$ of Chinese GWG distributions. ${ }^{36}$ In a recently published follow-up study including 34,288 pregnancies, ${ }^{37}$ the author managed to develop GWG charts of Chinese women based on the same BMI classification system in our study, and found that the IOM recommended ranges fell within the 25th and 75th percentiles for underweight and normal weight women but fell below the 50th percentile for overweight and obese women. Neither aforementioned studies included data relating to postpartum outcomes. Elevating the upper limit of the GWG ranges may lead to long-term adverse outcomes, including postpartum weight retention childhood obesity, and adolescent cardiometabolic risk. 38,39,40,41 Nonetheless, a meta-analysis of Westerners from 25 pooled cohort studies including 196,670 participants revealed that GWG ranges associated with the lowest risks for any adverse outcome were roughly comparable with the IOM ranges for underweight, normal weight, and overweight, and were lower for all obesity grades. ${ }^{42}$

A cohort study, the INTERGROWTH-21st Project, ${ }^{43}$ reported that the weight gain pattern in normal weight women who came from eight geographically diverse urban areas, including China, was strikingly similar, indicates that separate GWG charts for women from different racial groups are not necessary. The researchers speculating that the racial differences reported are more likely caused by social economical or lifestyle factors than true biological differences in nutrient absorption among uncomplicated women. ${ }^{44,45,46}$ Until now, the IOM recommendations was well-accepted in China, Our data showed that GWG within IOM recommendations may help prevent various adverse perinatal outcomes. Given the risks of a GWG outside of the IOM recommendations, as documented in this population based study whereby only $44.1 \%$ of women achieved a optimal GWG targets. Appropriate management of GWG, by way of lifestyle instructions in diet, exercise and other health behaviours, ${ }^{47}$ will assists in promoting maternal and neonatal health.

This study has several limitations. First, the analyses did not account for potential confounding factors, such as medical insurance type or hospital type. Second, first trimester pregnancy BMI, instead of pre-pregnancy $\mathrm{BMI}$, was used to classify women. This introduces the possibility of mis-classification bias. We did not assume that women gained a standard weight during first trimester which ignored individual difference. 
However, it is not feasible to measure the pre-pregnancy weight in clinical practise and self-reported prepregnancy weight is generally unreliable. Noted strengths included prospectively designed and well defined net-work based data acquisition system with detailed information on perinatal outcomes. The sample size was the largest concerning Chinese ethics before submission and spanned the four main ecnomic zones of China and is representative. With rigorous ascertainment of outcomes and calculation of GWG to account for the duration of pregnancy. The results presented as incidence rates and adjusted odds ratio is readily for clinical counseling.

\section{Conclusion}

Inadequate and excessive GWG both remained to be a big issue in Chinese pregnant population, IOM guidelines is applicable in predicting adverse outcomes and maintaining appropriate GWG is essential in preventing adverse maternal and neonatal outcomes.

\section{Declarations}

\section{Acknowledgements}

We thank the following hospitals, including Beijing Haidian maternal and child health hospital, Sichuan University West China Second Hospital, Southern Medical University Nanfang Hospital, Northwest women and children's hospital, Ruijin maternal and child health hospital, Hunan maternal and child health care hospital, and Shengjing Hospital affiliated to China Medical University, where the register work was conducted for this program.

\section{Details of ethics approval}

Our study was performed in compliance with the Declaration of Helsinki (as revised in 2013). The retrospective birth registry study protocol was approved by the Ethics Committee of Peking Union Medical College Hospital (reference number PUMCH-JS-1151) under a waiver of informed consent from individual patients.

\section{Contributions}

JS Gao conceived the study design which was advised by JT Liu; XT Wang, J He, JX Sun, XW Liu and SX Liao helped to collect data and supervise the collection process; Hu J wrote statistical program and Liu S analyzed the data; Dong $\mathrm{J}$ was responsible for designing the network based system with manuscripts contrived by JS Gao and JT Liu; Hu J edited the draft and had primary responsibility for the final content. Gao JS and Liu JT critically reviewed the draft and provided important intellectual content to the interpretation of the results. All the authors read and approved the final manuscript.

\section{Corresponding author}

Correspondence to Gao Jinsong and Liu Juntao

Funding information

Page 13/19 
This study was supported by the 13th Five-Year National Science and Technology Support Program (number [no.] 2015BAI13B04) and CAMS Innovation Fund for Medical Science (no. 2017-I2M-3-007).

\section{References}

1. Goldstein RF. et al. Association of GWG With Maternal and Infant Outcomes: A Systematic Review and Meta-analysis. JAMA. 2017 Jun 6;317(21):2207-2225.

2. Kominiarek MA, Peaceman AM. GWG. Am J Obstet Gynecol. 2017 Dec;217(6):642-651.

3. Li C, Liu Y, Zhang W. Joint and Independent Associations of GWG and Pre-Pregnancy Body Mass Index with Outcomes of Pregnancy in Chinese Women: A Retrospective Cohort Study. PLoS One. 2015 Aug 27;10(8):e0136850.

4. Melby, M.K., Yamada, G. and Surkan, P.J. Inadequate GWG increases risk of small-for-gestational-age term birth in girls in Japan: A population-based cohort study. J. Hum. Biol. 28: 714-720.

5. Edwards LE, Hellerstedt WL, Alton IR, Story M, Himes JH. Pregnancy complications and birth outcomes in obese and normal-weight women: effects of gestational weight change. Obstet Gynecol 1996;87:38994.

6. Kabiru W, Denise Raynor B. Obstetric outcomes associated with increase in BMI category during pregnancy. Am J Obstet Gynecol 2004;191:928-32.

7. Hilson JA, Rasmussen KM, Kjolhede CL. Nutrient Requirements and Optimal Nutrition Excessive Weight Gain during Pregnancy Is Associated with Earlier Termination of breast feeding among white women. $J$ Nutr 2006;136:140-6.

8. Baker JL, Michaelsen KF, Sorensen TIA, Rasmussen KM. High prepregnant body mass index is associated with early termination of full and any breastfeeding in Danish women. Am J Clin Nutr 2007;86:404-11.

9. Tong M.et al. Association between GWG and exclusive breast-feeding for the first 6 months postpartum in Chinese women. Public Health Nutr. 2019 Aug;22(11):2092-2098.

10. Saldana TM, et al. The relationship between pregnancy weight gain and glucose tolerance status among black and white women in central North Carolina. Am J Obstet Gynecol 2006;195:1629-35.

11. Sanabria-Martínez G, et al. Effectiveness of physical activity interventions on preventing gestational diabetes mellitus and excessive maternal weight gain: a meta-analysis. BJOG. 2015 Aug;122(9):1167-74.

12. Sibai BM. et al. Risk factors associated with preeclampsia in healthy nulliparous women. Am J Obstet Gynecol 1997;177:1003-10.

13. Bodnar LM, Catov JM, Klebanoff MA, Ness RB, Roberts JM. Prepregnancy body mass index and the occurrence of severe hypertensive disorders of pregnancy. Epidemiology 2007;18:234-9.

14. Zhuang C. et al. Risk factors and potential protective factors of pregnancy-induced hypertension in China: A cross-sectional study. J Clin Hypertens (Greenwich). 2019 May;21(5):618-623.

15. Sherrard A. et al. Maternal anthropometric risk factors for caesarean delivery before or after onset of labour. BJOG 2007;114:1088-96. 
16. Kominiarek, M.A.,Saade, G.,Mele, L ,et al. Association Between GWG and Perinatal Outcomes. Obstetrics \& Gynecology, 2018. 132(4): 875-881.

17. Institute of Medicine (IOM-US) Committee on Nutritional Status During Pregnancy and Lactation. Nutrition during pregnancy.National Academies Press, 1990.

18. IOM (Institute of Medicine)/NRC (National Research Council). Weight gain during pregnancy: reexamining the guidelines. In: Rasmussen $\mathrm{KM}$, Yaktine $\mathrm{AL}$, eds. Food and Nutrition Board and Board on Children, Youth, and Families. National Academies Press (US), 2009.

19. Parker JD, Abrams B. Prenatal weight gain advice: an examination of the recent prenatal weight gain recommendations of the Institute of Medicine. Obstet Gynecol. 1992;79:664e669.

20. Edwards LE, Hellerstedt WL, Alton IR, Story M, Himes JH. Pregnancy complications and birth outcomes in obese and normal-weight women: effects of gestational weight change. Obstet Gynecol. 1996;87:389e394.

21. The Use and Interpretation of Anthropometry. Physical status: The use and interpretation of anthropometry. Report of a WHO expert committee. World Health Organ Tech Rep Ser 1995; 854: 1-452.

22. Obesity. Preventing and managing the global epidemic: Report of a WHO Consultation on Obesity, Geneva. 3. Global prevalence and secular trends in obesity. Geneva: World Health Organization, 1997.

23. Obstetrics Subgroup, Chinese Society of Obstetrics and Gynecology, Chinese Medical Association Guideline of preconception and prenatal care (2018) (Article in Chinese) Zhonghua Fu Chan Ke Za Zhi. 2018;53(1):7-13.

24. Yang YD, Yang HX. Investigation into the clinical suitability of IOM 2009 guidelines regarding weight gain during pregnancy for women with full term singleton fetus in China(Article in Chinese). Zhonghua Fu Chan Ke Za Zhi. 2012 Sep;47(9):646-50.

25. Choo V. WHO reassesses appropriate body-mass index for Asian populations. Lancet. 2002;360:235.

26. Xi B, Liang Y, He T, et al. Secular trends in the prevalence of general and abdominal obesity among Chinese adults, 1993-2009. Obes Rev. 2012;13:287-296.

27. Zhou BF, et al; Cooperative Meta-Analysis Group of the Working Group on Obesity in China. Predictive values of body mass index and waist circumference for risk factors of certain related diseases in Chinese adults-study on optimal cut-off points of body mass index and waist circumference in Chinese adults. Biomed Environ Sci. 2002 Mar;15(1):83-96.

28. Diagnostic criteria and classification of hyperglycaemia first detected in pregnancy: a World Health Organization Guideline. Diabetes Res Clin Pract. 2014 Mar;103(3):341-63.

29. Dai L, et al. Birth weight reference percentiles for Chinese. PLoS One. 2014;9(8):e104779. doi: 10.1371/journal.pone.0104779.

30. Budde MP, et al. Risk factors for placental abruption in a socio-economically disadvantaged region. $J$ Matern Fetal Neonatal Med. 2007 Sep;20(9):687-93.

31. Carnero AM, Mejía CR, García PJ. Rate of gestational weight gain, pre-pregnancy body mass index and preterm birth subtypes: a retrospective cohort study from Peru. BJOG. 2012;119:924-35.

32. Nohr EA, et al. Obesity, gestational weight gain and preterm birth: a study within the Danish National Birth Cohort. Paediatr. Perinat. Epidemiol. 2007;21:5-14. 
33. Oken E, Kleinman KP, Belfort MB, Hammitt JK, Gillman MW. Associations of gestational weight gain with short-and longer-term maternal and child health outcomes. Am. J. Epidemiol. 2009;170:173-80.

34. Pigatti SF, et al. Role of Body Mass Index and gestational weight gain on preterm birth and adverse perinatal outcomes. Sci Rep. 2019;9:13093.

35. Zhou YB, et al. Gestational weight gain, cesarean delivery, and cesarean delivery on maternal request: a cohort analysis of Chinese nulliparous women. Ann Epidemiol. 2017;27(2):96-102.e3.

36. Wong W, Tang NL, Lau TK, Wong TW. A new recommendation for maternal weight gain in Chinese women. J Am Diet Assoc. 2000;100(7):791-796.

37. Huang $X$, et al. Gestational weight gain in Chinese women - results from a retrospective cohort in Changsha, China. BMC Pregnancy Childbirth. 2018 May 29;18(1):185.

38. Mannan M,et al. Association between weight gain during pregnancy and postpartum weight retention and obesity: a bias-adjusted meta-analysis. Nutr Rev. 2013;71(6):343-352.

39. Rong K, et al. Pre-pregnancy BMI, gestational weight gain and postpartum weight retention: a metaanalysis of observational studies. Public Health Nutr. 2015;18(12):2172-2182.

40. Gaillard R, et al. Associations of maternal prepregnancy body mass index and gestational weight gain with cardio-metabolic risk factors in adolescent offspring: a prospective cohort study. BJOG. 2016;123(2):207-216.

41. Lau EY, et al. Maternal weight gain in pregnancy and risk of obesity among offspring: a systematic review. J Obes. 2014;2014:524939.

42. LifeCycle Project-Maternal Obesity and Childhood Outcomes Study Group. Association of Gestational Weight Gain With Adverse Maternal and Infant Outcomes. JAMA. 2019;321(17):1702-1715.

43. Cheikh Ismail L, et al. Gestational weight gain standards based on women enrolled in the Fetal Growth Longitudinal Study of the INTERGROWTH-21st Project: a prospective longitudinal cohort study. BMJ. 2016 Feb 29;352:i555.

44. Caulfield LE, Witter FR, Stoltzfus RJ. Determinants of gestational weight gain outside the recommended ranges among black and white women. Obstet Gynecol 1996;87:760-6.

45. Chu SY, et al. Gestational weight gain by body mass index among US women delivering live births, 20042005: fueling future obesity. Am J Obstet Gynecol 2009;200:271.e1-7. 10.1016/j.ajog.2008.09.879. 19136091.

46. Yang W, et al. Relationship Between Gestational Weight Gain and Pregnancy Complications or Delivery Outcome. Sci Rep. 2017 Oct 2;7(1):12531.

47. Muktabhant B, et al. Diet or exercise, or both, for preventing excessive weight gain in pregnancy. Cochrane Database Syst Rev 2015;6:CD007145.

\section{Figures}




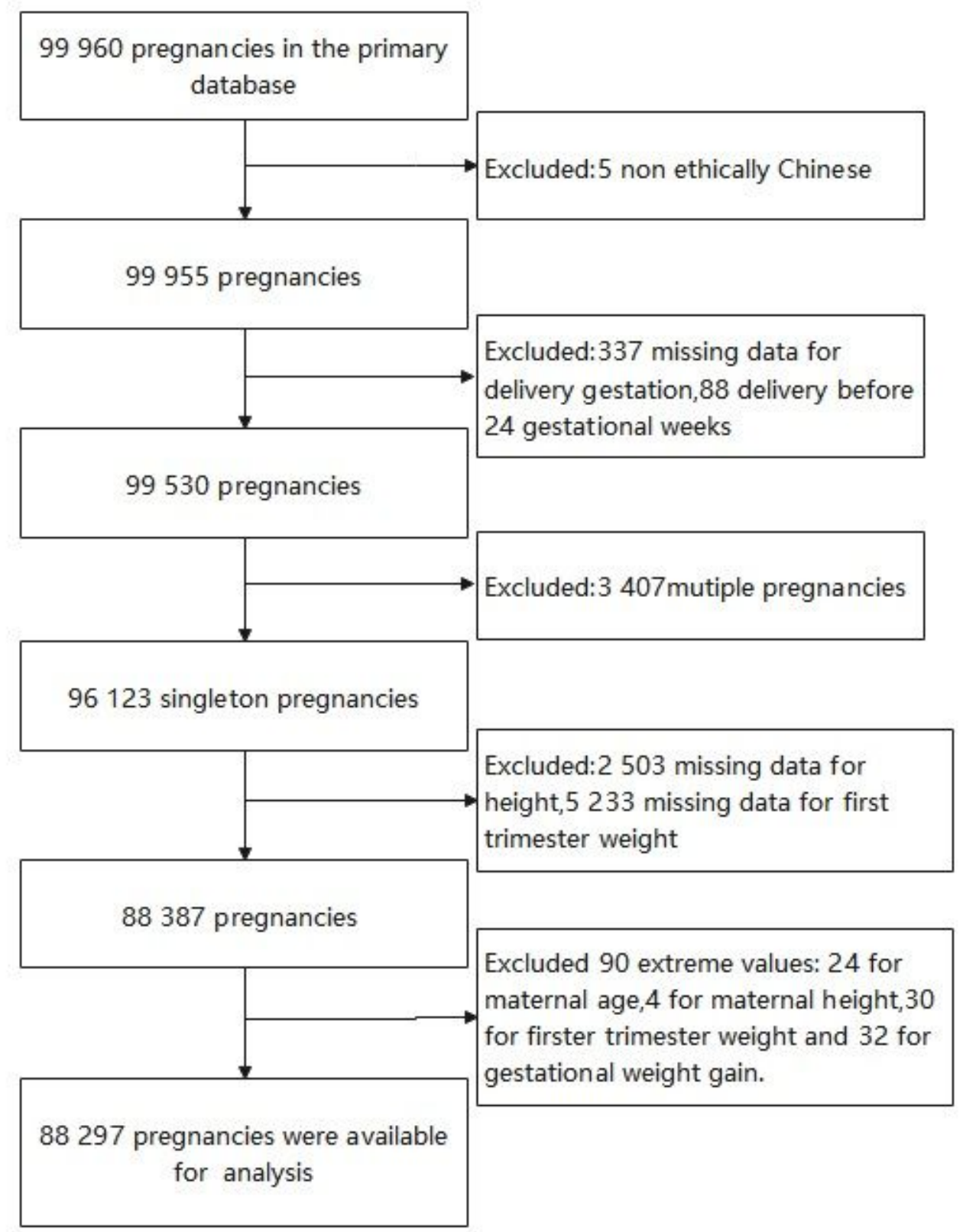

Figure 1

Flow chart for data selection process 

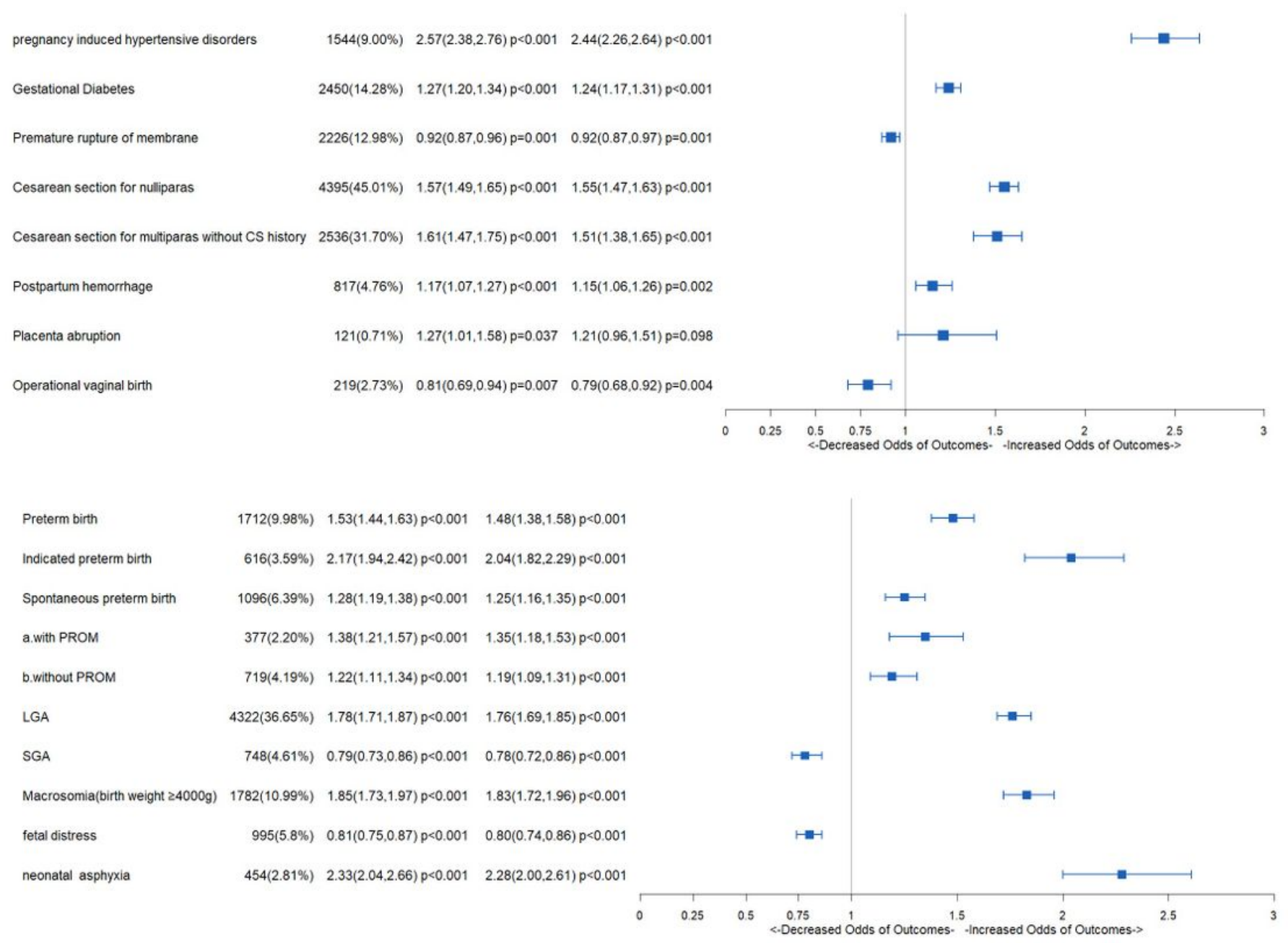

\section{Figure 2}

a. Forest plot for adjusted OR of GWG above recommendations and maternal outcomes. Abbreviations: CS, cesarean section. Reference group: GWG within recommendations. b. Forest plot for adjusted OR of GWG above recommendations and neonatal outcomes. Abbreviations: PROM, premature rupture of fetal membrane; LGA, large for gestational age; SGA, small for gestational age.Reference group: GWG within recommendations. 


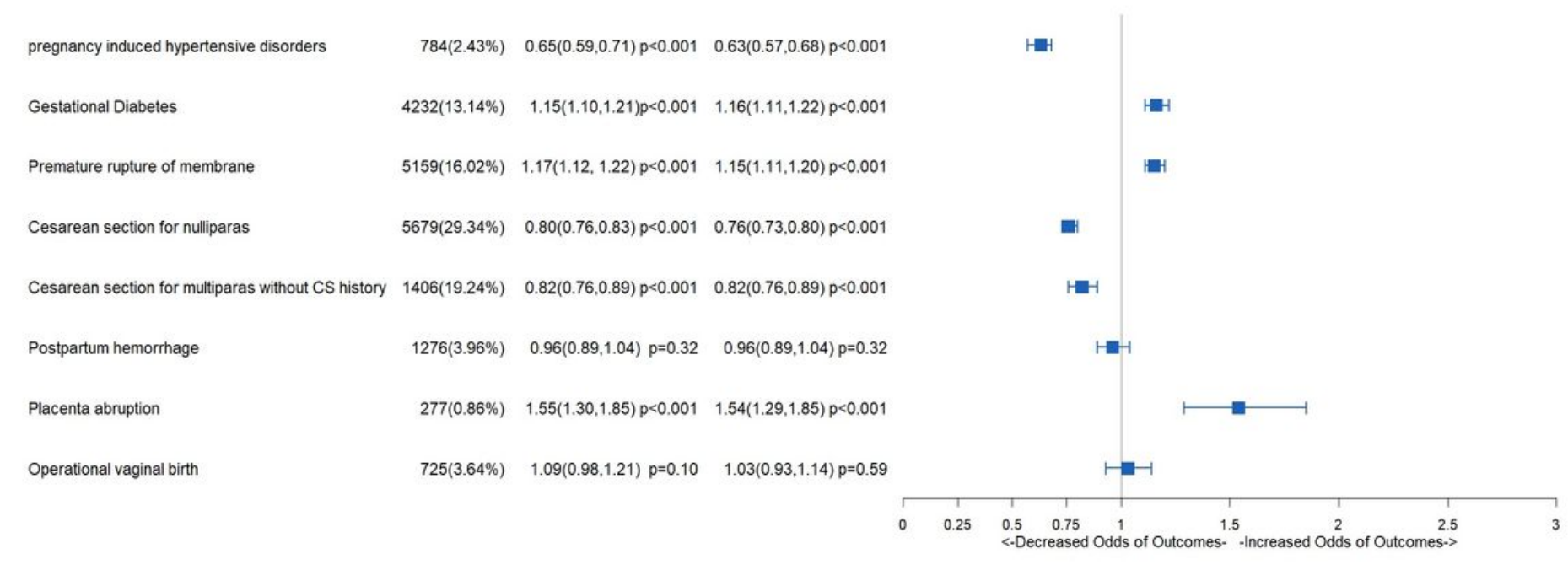

$\begin{array}{lrrr}\begin{array}{l}\text { Preterm birth } \\ \text { Indicated preterm birth }\end{array} & 670(2.08 \%) & 1.24(1.11,1.38) p<0.001 & 1.27(1.14,1.42) p<0.001 \\ \text { Spontaneous preterm birth } & 1931(5.99 \%) & 1.20(1.12,1.28) p<0.001 & 1.19(1.11,1.27) p<0.001 \\ \text { a.with PROM } & 711(2.21 \%) & 1.39(1.24,1.54) p<0.001 & 1.35(1.21,1.50) p<0.001 \\ \text { b. without PROM } & 1220(3.79 \%) & 1.10(1.02,1.19) p=0.017 & 1.10(1.02,1.20) p=0.016 \\ \text { LGA } & 3461(11.23 \%) & 0.62(0.59,0.65) p<0.001 & 0.61(0.59,0.64) p<0.001 \\ \text { SGA } & 2619(8.50 \%) & 1.52(1.43,1.61) p<0.001 & 1.50(1.41,1.60) p<0.001 \\ \text { Macrosomia(birth weight } \geq 4000 g) & 1066(3.46 \%) & 0.54(0.50,0.58) p<0.001 & 0.53(0.49,0.57) p<0.001 \\ \text { fetal distress } & 2739(8.5 \%) & 1.22(1.16,1.29) p<0.001 & 1.19(1.12,1.26) p<0.001 \\ \text { neonatal asphyxia } & 530(1.73 \%) & 1.42(1.25,1.61) p<0.001 & 1.42(1.25,1.61) p<0.001\end{array}$

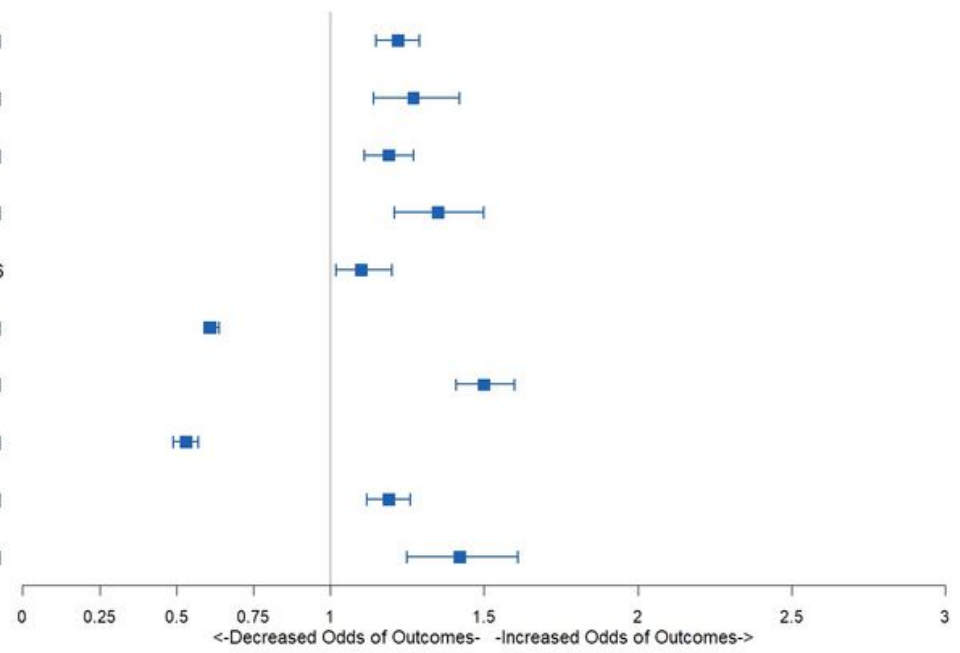

\section{Figure 3}

a. Forest plot for adjusted OR of GWG below recommendations and maternal outcomes. Abbreviations: CS, cesarean section. Reference group: GWG within recommendations. b. Forest plot for adjusted OR of GWG below recommendations and neonatal.Abbreviations: PROM, premature rupture of fetal membrane; LGA, large for gestational age; SGA, small for gestational age Reference group: GWG within recommendations. 